\title{
RESÍDUOS AGROINDUSTRIAIS PRENSADOS COMO SUBSTRATO PARA A PRODUÇÃO DE MUDAS DE CANA-DE-AÇÚCAR
}

\author{
Ivan Ferrreira Morgado; ; José Geraldo de Araujo Carneiro; Paulo Sérgio dos Santos Leles; \\ Deborah Guerra Barroso $2,4 *$ \\ ${ }^{1}$ Depto. de Fitotecnia - UFRRJ, Est. do Açúcar km $5 \mathrm{~s} / \mathrm{n}^{\circ}$ - CEP: 28020-560 - Campos dos Goytacazes, RJ. \\ ${ }_{3}^{2}$ Setor de Silvicultura - CCTA/LFIT/UENF, Av. Alberto Lamego, 2000 - CEP: 28015-620 - Campos dos Goytacazes, RJ. \\ ${ }^{3}$ Instituto de Florestas - UFRRJ, Antiga Estrada Rio-SP, km 47 - CEP: 23890-000 - Seropédica, RJ. \\ ${ }^{4}$ Bolsista FAPERJ. \\ *Autor correspondente <deborah@uenf.br>
}

\begin{abstract}
RESUMO: Um experimento com Saccharum spp. foi conduzido, tendo como objetivo desenvolver uma nova metodologia de produção de mudas desta espécie, utilizando três resíduos agroindustriais prensados, como substrato. Foram estudadas diferentes proporções de bagaço de cana-de-açúcar, torta de filtro de usina e casca de coco na composição do substrato. O sistema de blocos prensados, de origem finlandesa, foi comparado ao sistema convencional. A qualidade das mudas, no final da rotação no viveiro, foi avaliada através do diâmetro de colo, número de perfilhos por planta, peso de matéria seca das partes aérea, radicular e total e o desempenho das mudas 12 meses após o plantio, através do comprimento, diâmetro, número de entrenós e peso dos colmos. Os três resíduos mostraram condições de serem utilizados em mistura para o preparo de blocos prensados. Os blocos prensados de origem finlandesa e os preparados com bagaço de cana-de-açúcar e torta de filtro de usina, misturados, respectivamente, nas proporções de 70 e $30 \%, \mathrm{v} \mathrm{v}^{-1}$, foram os mais adequados para a produção de mudas de Saccharum spp.. As médias dos parâmetros morfológicos das mudas produzidas pelo sistema utilizado pelosistema convencional foram classificadas entre as mais baixas do experimento. Palavras-chave: cana-de-açúcar, substrato, recipiente, muda
\end{abstract}

\section{PRESSED INDUSTRIAL RESIDUES AS SUBSTRATE FOR SUGAR CANE SEEDLING PRODUCTION}

\begin{abstract}
An experiment with Saccharum spp. was carried out to develop a new methodology for plantlet production using pressed industrial residues as substrate. The proportions of sugar cane bagasse, sugar plant filter cake and coconut husk in the substrate composition were analyzed along with the advantages of the Finland pressed block and the conventional systems used for plantlet production. The seedlings quality at the and of the plant propagation stage was evaluated through plantlet diameter and height, shoot and root, total dry weights, and performance at the twelfth month. Selection for planting was based on stem length, diameter, quantity and weight. The three residues tested were suitable to be used in a mixture to prepare pressed blocks. Imported blocks and the ones prepared with both, sugar cane bagasse and the sugar plant filter cake mixed, 70 and $30 \%, v^{-1}$ respectively, were the most suitable. The average morphological parameters of the plantlets produced by the conventional system were classified among the lowest.
\end{abstract}

Key words: sugar cane, substrate, container, seedling

\section{INTRODUÇÃO}

A situação atual da economia brasileira sugere esforços na busca de sistemas mais produtivos e a custos mais baixos. $\mathrm{Na}$ atividade canavieira a produção de mudas com elevado padrão de qualidade constitui-se numa fase importante do processo produtivo. A implantação do Programa Nacional do Álcool (PROÁLCOOL) levou o governo e a iniciativa privada a investirem maciçamente no desenvolvimento de programas de melhoramento da cana-de-açúcar.

Devido à importância que os programas de melhoramento de cana-de-açúcar representam para o setor primário brasileiro, justifica-se a condução de pesquisas visando à qualidade de substrato para a produção de mudas mais vigorosas, mais resistentes às adversidades climáticas e a custos mais baixos.
A qualidade das mudas é fundamental, pois ela influencia na percentagem de sobrevivência, na velocidade de crescimento e na produção final. Além disso, mudas de melhor qualidade, por terem maior potencial de crescimento, exercem um melhor controle da vegetação invasora, reduzindo os custos dos tratos culturais.

Sanguino (1986) ressaltou a importância da qualidade das mudas na formação das lavouras. Alertou que a utilização de canaviais como fonte de mudas facilita a propagação de doenças e reduz a vida útil dos canaviais, causando grandes prejuízos.

Um dos problemas das mudas produzidas em recipientes são as deformações radiculares. O método de produção em blocos preparados com material prensado apresenta, como principais vantagens, a possibilidade de desenvolvimento do sistema radicular sem deformações, a facilidade de manuseio das mudas durante o plantio e a 
redução nos custos de produção, devido à possibilidade de uso intensivo da mecanização.

O sistema de blocos prensados é um processo difundido entre os países escandinavos, notadamente na Finlândia. Harstella et al. (1983) relataram que as primeiras pesquisas visando desenvolver um sistema de produção de mudas em blocos prensados foram iniciadas em 1979, na Estação Experimental de Suonenjoki, na Finlândia. Posteriormente, os trabalhos prosseguiram com a participação de uma empresa particular denominada VAPO.

Harstella et al. (1983), Parviainen \& Tervo (1989) apresentaram um processo constituído por blocos prensados de turfa seca $(60 \times 40 \mathrm{~cm})$, devidamente fertilizados. Não há limitações de paredes, proporcionando condições de desenvolvimento de raízes, por todos os lados, sem confinamento ou direcionamento. Após o período de rotação, por meio de uma máquina específica, os blocos são serrados através de um conjunto de serras circulares, introduzidas em sentidos transversais no bloco, passando em frestas das paredes laterais das caixas que contém os blocos. Desta forma, individualizam-se os torrões que são plantados com as mudas.

As pesquisas envolvendo o sistema de blocos prensados têm demonstrado que a poda do sistema radicular exerce um efeito positivo, aumentando a ramificação e o número de extremidades de novas raízes (Leles, 1998; Barroso, 1999).

Procurando materiais que pudessem apresentar desempenho equivalente aos blocos finlandeses, Carneiro \& Brito (1995) submeteram, isoladamente, xaxim moído, bagaço de cana triturado, "sphagnum" triturado, acícula seca triturada, fibras de celulose e vermiculita à prensagem após total encharcamento com água. A coesão dos blocos prensados úmidos foi observada após a secagem em estufa. Os autores concluíram que apenas xaxim moído, bagaço de cana triturado e "sphagnum" triturado deveriam ser selecionados para a confecção dos blocos.

$\mathrm{Na}$ Região Norte Fluminense, existem alguns resíduos da indústria canavieira, cujas características sugerem a possibilidade de constituírem substratos adequados para a produção de mudas de cana-de-açúcar.

O objetivo do trabalho foi desenvolver uma nova metodologia de produção de mudas de Saccharum spp., utilizando resíduos agroindustriais prensados como substrato, compará-la ao sistema convencional de produção de mudas desta espécie e determinar a adequação e as proporções ideais de cada um dos resíduos testados na composição do substrato.

\section{MATERIAL E MÉTODOS}

Esta pesquisa foi conduzida no Campus Dr. Leonel Miranda da UFRRJ, em Campos dos Goytacazes/RJ.

Na composição dos substratos, para a confecção dos blocos, foram utilizados três tipos de resíduos agroindustriais: bagaço de cana-de-açúcar, casca de coco e torta de filtro de usina, em diferentes proporções (TABELA1). O bloco finlandês, comercializado pela VAPO, é constituído por turfa seca fertilizada e prensada.

Estes resíduos foram devidamente preparados visando permitir que, após misturados e posteriormente prensados, formassem blocos coesos, em condições de permitir um desenvolvimento sem deformações radiculares das mudas até o final do período de rotação no viveiro.

Este preparo foi constituído por seis etapas seqüenciais: trituração, uniformização do comprimento das fibras, tratamento com fumigante, mistura dos materiais, prensagem e secagem dos blocos prensados.

O bagaço de cana-de-açúcar e a torta de filtro de usina utilizados encontravam-se em estágio intermediário de decomposição. A casca de coco estava seca e foi triturada em uma picadeira de ração para animais. Os materiais foram peneirados, utilizando-se malha de $1 \mathrm{~cm}$, fumigados e misturados em diferentes proporções, de forma homogênea, por meio de uma betoneira.

Foram preparadas caixas metálicas que serviram como fôrmas, com as seguintes dimensões: $60 \times 40 \times 20$ $\mathrm{cm}$ (comprimento $x$ largura $x$ altura).

Após o processamento das operações descritas, os substratos foram umedecidos, colocados em uma caixa metálica e submetidos a diferentes níveis de carga, em uma prensa hidráulica visando padronizar a altura final do bloco em $10 \mathrm{~cm}$. Sobre os blocos, durante a prensagem, foi colocada uma placa, tendo em sua face inferior cones com $1 \mathrm{~cm}$ de altura, destinados a marcar os orifícios para 0 transplantio dos explantes. Para a secagem os blocos ficaram expostos ao ar livre, durante 24 horas e, em seguida, colocados em estufa regulada a $60^{\circ} \mathrm{C}$, durante 48 horas.

Os blocos mantiveram coesão suficiente até o final do período de rotação no viveiro. Antes do plantio no campo utilizou-se uma lâmina de serra com dois cabos para a individualização das mudas.

Foram confeccionadas caixas de madeira para proteção de cada bloco prensado. Cada bloco recebeu 40 explantes espaçados de $8 \times 8 \mathrm{~cm}$, dispostos em cinco fileiras de oito explantes cada uma.

TABELA 1 - Percentuais dos materiais na composição volumétrica do substrato.

\begin{tabular}{cccc}
\hline Tratamentos & $\begin{array}{c}\text { Bagaço de } \\
\text { Cana }\end{array}$ & $\begin{array}{c}\text { Casca de } \\
\text { Coco }\end{array}$ & Torta de filtro \\
\hline 1 & 80 & 00 & 20 \\
2 & 80 & 20 & 00 \\
3 & 70 & 00 & 30 \\
4 & 60 & 00 & 40 \\
5 & 60 & 10 & 30 \\
6 & 60 & 30 & 10 \\
$7^{1 /}$ & \multicolumn{3}{c}{$\begin{array}{c}\text { Bloco } \\
\text { finlandes } \\
8^{2 /}\end{array}$} \\
\hline
\end{tabular}

"Blocos prensados de origem finlandesa.

2/ Sistema adotado pela UFRRJ. 
O sistema da UFRRJ foi adotado como testemunha. Foram utilizados explantes da variedade CB 45-3 produzidos na biofábrica do campus Dr. Leonel Miranda da UFRRJ. Os explantes foram transplantados para moldes de isopor medindo $58 \times 33 \times 6 \mathrm{~cm}$, contendo 112 cavidades afuniladas, em forma de pirâmide invertida, com o volume de $40 \mathrm{~cm}^{3}$ cada uma. Cada um destes moldes de isopor constituiu uma repetição. Utilizou-se, como substrato, uma mistura, em proporções volumétricas iguais, de bagaço de cana e torta de filtro de usina, previamente submetidos a um processo de fermentação aeróbica.

$\mathrm{Na}$ fase de viveiro, o delineamento experimental foi inteiramente casualisado, constituído por oito tratamentos e quatro repetições. Cada repetição foi composta por dois blocos prensados contendo 40 mudas cada uma. Cem dias após o transplantio dos explantes, foram retiradas quatro mudas aleatoriamente, em cada parcela, para a avaliação do peso de matéria seca da parte aérea, sistema radicular e total. Os dados foram submetidos à análise de variância e, em seguida, as médias comparadas pelo teste de Duncan, ao nível de $5 \%$.

$\mathrm{Na}$ fase de campo, as mudas foram plantadas em blocos casualizados, mantendo-se os mesmos tratamentos e número de repetições da fase de viveiro. Cada parcela experimental foi constituída por 36 mudas, dispostas em 3 fileiras de 4,80 m de comprimento espaçadas de 1,40 $\mathrm{m}$ entre si. Dentro das fileiras, o espaçamento entre as mudas foi de $0,40 \mathrm{~m}$.

Ao completar 12 meses após o plantio, o experimento foi colhido, sendo retirados 12 colmos, aleatoriamente, em cada parcela, quanto ao comprimento, diâmetro, número de entrenós e peso dos colmos.

\section{RESULTADOS E DISCUSSÃO}

A TABELA 2 apresenta as médias de peso de matéria seca mudas de Saccharum spp., no final da fase de viveiro.

TABELA 2 - Médias dos pesos de matéria seca da raiz (PSR), da parte aérea (PSA) e do total (PST), das mudas de Saccharum spp., aos 100 dias após o transplantio dos explantes para os substratos.

\begin{tabular}{cccc}
\hline Tratamento & \multicolumn{1}{c}{ PSR } & \multicolumn{1}{c}{ PSA } & PST \\
\hline & & - & - \\
1 & $24,33 \mathrm{bcd}$ & $30,45 \mathrm{c}$ & $54,78 \mathrm{~cd}$ \\
2 & $20,40 \mathrm{~cd}$ & $21,30 \mathrm{~d}$ & $41,70 \mathrm{de}$ \\
3 & $44,40 \mathrm{a}$ & $62,07 \mathrm{a}$ & $106,47 \mathrm{a}$ \\
4 & $32,28 \mathrm{bc}$ & $30,10 \mathrm{c}$ & $62,38 \mathrm{bc}$ \\
5 & $23,20 \mathrm{bcd}$ & $41,12 \mathrm{~b}$ & $64,32 \mathrm{bc}$ \\
6 & $27,58 \mathrm{bc}$ & $36,97 \mathrm{bc}$ & $64,55 \mathrm{bc}$ \\
7 & $34,38 \mathrm{ab}$ & $44,62 \mathrm{~b}$ & $79,00 \mathrm{~b}$ \\
8 & $14,25 \mathrm{~d}$ & $14,00 \mathrm{~d}$ & $28,25 \mathrm{e}$
\end{tabular}

Para cada característica analisada, médias seguidas pela mesma letra não diferem pelo teste de Duncan $(P<0,05)$.
Os tratamentos $3(70 \%$ de bagaço de cana $+30 \%$ de torta de filtro) e 7 (blocos de origem finlandesa) apresentaram as maiores médias de peso de matéria seca da raiz, não diferindo entre si.

Com relação ao peso de matéria seca da parte aérea, a média apresentada pelo tratamento 3 foi superior aos demais. As médias mais baixas do experimento foram observadas nos tratamentos $2(80 \%$ de bagaço de cana $+20 \%$ de casca de coco) e 8 (sistema adotado pela UFRRJ) que se equivaleram.

O peso de matéria seca da parte aérea é uma boa indicação da capacidade de resistência das mudas às condições adversas após o plantio (Schmidt-Vogt, 1966). Esta constatação foi confirmada também por Carneiro (1976).

Comparando-se as médias de peso de matéria seca total, verificou-se que o tratamento 3 também foi significativamente superior a todos os demais. As médias mais baixas do experimento foram observadas nos tratamentos 2 e 8 (sistema da UFRRJ), cujos valores equivaleram-se.

O fraco desempenho do tratamento 2 pode ser atribuído à participação excessiva do bagaço de cana e à ausência da torta de filtro na composição do substrato, tornando-o física e quimicamente inferior a outros. Isto ocorreu porque, entre os três resíduos, o bagaço de cana é o mais pobre em nutrientes (Morgado 1998). No caso do sistema adotado pela UFRRJ, acredita-se que, além da composição, o pequeno volume de substrato destinado a cada muda tenha restringido seu crescimento.

$\mathrm{Na}$ TABELA 3 são apresentados os resultados das medições dos parâmetros morfológicos no final da fase de campo.

O peso dos colmos não diferiu entre os tratamentos. Foram constatadas poucas diferenças entre os tratamentos para o comprimento, 0 diâmetro e 0 número de entrenós dos colmos.

TABELA 3 - Médias do comprimento (C), do diâmetro (D), do número de entrenós $(E N)$ e do peso $(P)$ dos colmos de Saccharum spp., aos 12 meses após o plantio.

\begin{tabular}{ccccc}
\hline Tratamento & $\mathrm{C}$ & $\mathrm{D}$ & $\mathrm{EN}$ & $\mathrm{P}$ \\
\hline & $\mathrm{m}$ & $\mathrm{mm}$ & & $\mathrm{kg}$ \\
1 & $2,02 \mathrm{a}$ & $26,16 \mathrm{c}$ & $17,00 \mathrm{ab}$ & $1,08 \mathrm{a}$ \\
2 & $1,68 \mathrm{~b}$ & $28,17 \mathrm{a}$ & $15,50 \mathrm{~b}$ & $1,02 \mathrm{a}$ \\
3 & $1,95 \mathrm{ab}$ & $27,54 \mathrm{ab}$ & $17,00 \mathrm{ab}$ & $1,17 \mathrm{a}$ \\
4 & $1,98 \mathrm{ab}$ & $27,59 \mathrm{ab}$ & $17,00 \mathrm{ab}$ & $1,15 \mathrm{a}$ \\
5 & $1,93 \mathrm{ab}$ & $26,65 \mathrm{bc}$ & $17,75 \mathrm{a}$ & $1,07 \mathrm{a}$ \\
6 & $1,90 \mathrm{ab}$ & $26,92 \mathrm{bc}$ & $16,50 \mathrm{ab}$ & $1,00 \mathrm{a}$ \\
7 & $1,89 \mathrm{ab}$ & $25,81 \mathrm{c}$ & $17,50 \mathrm{a}$ & $1,02 \mathrm{a}$ \\
8 & $1,91 \mathrm{ab}$ & $27,39 \mathrm{ab}$ & $17,25 \mathrm{a}$ & $1,10 \mathrm{a}$ \\
\hline
\end{tabular}

Para cada característica analisada, médias seguidas pela mesma letra não diferem pelo teste de Duncan $(P<0,05)$. 
Os tratamentos $3(70 \%$ de bagaço de cana $+30 \%$ de torta de filtro) e 7 (blocos de origem finlandesa) mantiveram-se superiores aos demais, confirmando no campo o comportamento observado no viveiro.

Entretanto, houve recuperação dos tratamentos com desempenho mais fraco na fase de viveiro, tendo em vista que, a maioria dos parâmetros morfológicos, avaliados na fase final de campo, apresentaram resultados equivalentes.

\section{CONCLUSÕES}

- O bagaço de cana-de-açúcar, a casca de coco e a torta de filtro de usina mostraram possibilidades de uso em mistura para o preparo de blocos prensados para a produção de mudas de Saccharum spp.

- Dentre os três resíduos testados, a casca de coco foi o que apresentou menor adequação à produção de mudas. À medida em que aumentou a sua participação no substrato, observou-se uma tendência à obtenção de valores mais baixos dos parâmetros morfológicos.

- O sistema de blocos prensados finlandeses e o de blocos preparados com bagaço de cana-de-açúcar e torta de filtro de usina, misturados, respectivamente, nas proporções de 70 e $30 \%, v^{-1}$, foram os mais adequados para a produção de mudas de Saccharum spp.

\section{REFERÊNCIAS BIBLIOGRÁFICAS}

BARROSO, D.G. Qualidade de mudas de Eucalyptus camaldulensis e E. urophylla produzidas em tubetes e em blocos prensados com diferentes substratos. Campos dos Goytacazes, 1999. 72p. Tese (Doutorado) - Universidade Estadual do Norte Fluminense.
CARNEIRO, J.G. de A. Determinação do padrão de qualidade de mudas de Pinus taeda L. para plantio definitivo. Curitiba, 1976. 70p. Dissertação (Mestrado) - Universidade Federal do Paraná.

CARNEIRO, J.G. de A.; BRITO, M.A.R. Nova metodologia para produção mecanizada de mudas de Pinus taeda $L$. em recipientes com raízes laterais podadas. Revista Árvore, v.22, p.63-77, 1995.

HARSTELLA, P.; PARVIAINEN, J.; TERVO, L. Production of containerized seedlings by "box- pruning"- a description of the method and nursing experiences, In: SEMINAR ON MACHINES AND TECHNIQUES FOR FOREST PLANT PRODUCTION, Tratanska Lomnica, Czechoslovakia, 1983. p.1-7.

LELES , P.S. dos S. Produção de mudas de Eucalyptus camaldulensis, E. grandis e E. pellita produzidas em blocos prensados e em tubetes. Campos dos Goytacazes, 1998. 70p. Tese (Doutorado) - Universidade Estadual do Norte Fluminense.

MORGADO, I.F. Resíduos agroindustriais prensados como substrato para a produção de mudas de Eucalyptus grandis Hill ex Maiden e Saccharum spp. Campos dos Goytacazes, 1998. 102p. Tese (Doutorado) - Universidade Estadual do Norte Fluminense.

PARVIAINEN, J.V.; TERVO, L. A new approach for production of containerized coniferous seedlings using peat sheets coupled with root pruning. Forestry Supplement, v.62, p.87-94, 1989.

SANGUINO, A. Como formar viveiros de mudas de cana-deaçúcar. In: SEMINÁRIO DE TECNOLOGIA AGRONÔMICA, 3., Piracicaba, 1986. Anais. Piracicaba: ESALQ/USP, 1986. p.229-242.

SCHMIDT-VOGT, $H$. Wachstum und qualitaet von forstpflanzen. 2.ed. Munique: Bayerischer Landwirtschaftverlag, 1966. 210p.

Recebido em 16.11 .99 